\title{
LEVANTAMENTO DA COMUNIDADE DE AVES EM UM SISTEMA DE FRAGMENTOS FLORESTAIS INTERCONECTADOS POR CORREDORES ECOLÓGICOS NO MUNICÍPIO DE LAVRAS - MINAS GERAIS
}

\author{
Bruno Senna Corrêa - Instituto Federal de Educação, Ciência e Tecnologia do Sul de Minas Gerais - \\ Campus Inconfidentes, Praça Tiradentes, 416 - Centro - CEP 37576-000, Inconfidentes, MG - Brazil; \\ bruno.senna@gmail.com \\ Aloysio Souza Moura - Aluno de graduação do curso de Ciências Biológicas do Centro Universitário de Lavras - \\ UNILAVRAS
}

\section{Bird community survey in forest fragments connected by ecological corridors in Lavras c municipality - Minas Gerais-Brazil}

\section{RESUMO}

Foi realizado um levantamento da comunidade de aves em fragmentos florestais conectados por corredores ecológicos, no município de Lavras, MG - Brasil, compreendido entre as coordenadas $21^{\circ} 17^{\prime} 15.1^{\prime \prime} \mathrm{S}$ e $21^{\circ} 19^{\prime} 25.2^{\prime} \mathrm{S}, 44^{\circ} 58^{\prime} 59.3^{\prime \prime} \mathrm{W}$ e $44^{\circ} 59^{\prime} 53.1^{\prime \prime} \mathrm{W}$ e altitude média de $982 \mathrm{~m}$. Foram estudados oito fragmentos de floresta estacional semidecidual ( 1,0 ha a 12,1 ha) conectados por cinco corredores ecológicos de vegetação arbórea instalado sobre "valos". Objetivou-se neste trabalho analisar como propriedades da diversidade de aves estão relacionadas aos elementos da paisagem, em uma escala local. O método utilizado para registro de avifauna foi de visualização/ vocalização em pontos fixos distribuídos no interior e borda dos fragmentos, corredor e matriz. Foram registradas, em 132 dias (792 horas de observação), 179 espécies de aves, distribuídas em 44 famílias, sendo três espécies consideradas endêmicas do Cerrado, uma endêmica de Mata Atlântica, duas espécies ameaçadas de extinção no Brasil, duas espécies vulneráveis e duas introduzidas. Foram observadas diferenças na distribuição de aves entre fragmentos florestais, corredores ecológicos e matriz, sendo mais freqüentes espécies adaptadas à matriz (ambientes abertos) do que espécies florestais, entretanto não foram encontrados resultados significativos que demonstrassem relação da variação da riqueza e da abundância, entre os ambientes estudados. Os resultados evidenciam a relativa ineficiência de fragmentos florestais pequenos mesmo que conectados para a manutenção da diversidade biológica de espécies florestais.

Palavras-chave: Comunidade de aves, fragmentos florestais, corredores ecológicos, diversidade de espécies.

\begin{abstract}
It has been carried out a bird community survey in forest fragments connected by ecologi-

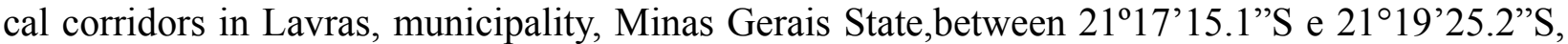
$44^{\circ} 58^{\prime} 59.3^{\prime \prime} \mathrm{W}$ e $44^{\circ} 59^{\prime} 53.1^{\prime \prime} \mathrm{W}$ coordinates and $982 \mathrm{~m}$ average altitude. Eight stational semi deciduous forest fragments ( 1,0 ha to 12,1 ha) connected by five ecological corridors have been studied. The aims of this work were analize how bird diversity characteristics were related to landscape elements in local scale. The used method for bird community survey was point sampling. It has been registered in 132 days (792 hours), 179 species, distributed in 44 families, 3 of which were endemic of Cerrado, 1 was endemic of Atlantic Forest, 2 were threatened in Brazil, 2 were vulnerable and 2 were introduced. Differences in bird distribution have been observed, between forest fragments and ecological corridors, being observed mostly frequent open areas species than forest species. This
\end{abstract}


work confirms the inefficience of small forest fragments even connected by ecological corridors to maintain bird forest species diversity. Key-word: Bird community, forest fragments, ecological corridors, species diversity.

\section{INTRODUÇÃO}

A fragmentação introduz uma série de novos fatores na história evolutiva de populações naturais de plantas e animais (Kellman, 1996; Groom \& Grubb, 2006). Essas mudanças afetam, de forma diferenciada, os parâmetros demográficos de mortalidade e natalidade de diferentes espécies e, portanto, a estrutura e dinâmica de ecossistemas (Noss et al., 2006). No caso de espécies arbóreas, a alteração na abundância de polinizadores, dispersores, predadores e patógenos, alteram as taxas de recrutamento de plântulas; e os incêndios e mudanças microclimáticas, que atingem de forma mais intensa as bordas dos fragmentos, alteram as taxas de mortalidade de árvores. As evidências científicas sobre esses processos têm se avolumado nos últimos anos (Laurance \& Bierregaard Junior, 1997; Groom \& Grubb, 2006; Noss et al., 2006; Wickam et al., 2007).

A composição das aves do bioma Cerrado responde a um gradiente vegetacional apresentado por esse bioma de tal modo que Silva (1995 a,b) reconhece três conjuntos de espécies, quanto ao uso do habitat: restritas a ambientes abertos, a ambientes florestais e semi-independentes.

Essa composição pode estar relacionada a alguns fatores, seja direta ou indiretamente. A degradação de habitat parece ser um importante fator na distribuição de avifauna do Cerrado. Essa degradação, em decorrência principalmente da expansão agrícola, parece favorecer algumas famílias de aves, enquanto outras se deslocam dessas áreas antropizadas, tornando-se restritas a áreas de refúgio (Marini, 2001).
Existem poucos estudos relacionados à estrutura da comunidade de avifauna, em paisagens fragmentadas de Cerrado (Tubelis, 1997; Lima et al., 2003; Penteado, 2006). Atualmente, observam-se vários estudos de avifauna relacionados a fragmentos e corredores de Mata Atlântica, no estado de São Paulo (Develey, 2004; Uezu et al., 2005; Antunes, 2005), Minas Gerais (Ribon, 2003; Maldonado-Coelho \& Marini, 2000), Bahia (Laps et al., 2003) e Paraná (Soares \& Anjos, 1999; Anjos, 2001; Anjos et al., 2004). Não se conhecem os parâmetros, direta e indiretamente relacionados à dinâmica de avifauna em fragmentos de florestas estacionais semideciduais inseridas no bioma Cerrado, nem tão pouco sobre os corredores que os conectam. Tais estudos podem fornecer subsídios para o desenvolvimento de planos de manejo e conservação de fauna e flora em paisagens fragmentadas de Cerrado, além de possibilitar a preservação de espécies endêmicas do bioma. Tendo em vista os fatores relacionados com a composição da avifauna em ambientes fragmentados e o pouco conhecimento existente em relação a paisagens de Cerrado fragmentadas pela atividade agropecuária, nesse trabalho objetivou-se principalmente, analisar como a paisagem e os habitats característicos dela estão associados com a riqueza de espécies de avifauna em uma escala de paisagem, na fazenda da Lage e áreas adjacentes, município de Lavras MG. Testou-se a seguinte hipótese: a distribuição da diversidade de aves é influenciada pelas características estruturais do sistema corredor/ fragmento/ matriz, partindo do pressuposto de que os diferentes elementos da paisagem oferecem atributos distintos à comunidade de aves ou à parte dela.

\section{MATERIAL E MÉTODOS}

\section{Área de estudo}

O sistema corredor-fragmento estudado está localizado na cidade de Lavras, re- 
gião do Alto Rio Grande, Sul de Minas Gerais e está compreendido entre as coordenadas $21^{\circ} 17^{\prime} 15.1^{\prime \prime} \mathrm{S}$ e $21^{\circ} 19^{\prime} 25.2^{\prime \prime} \mathrm{S}, 44^{\circ} 58^{\prime} 59.3^{\prime \prime}$ W e 4459'53.1'W (Figura 1). A área de estudo encontra-se a, aproximadamente, $6 \mathrm{~km}$ do município de Lavras, na encosta da Serra do Carrapato. Ao todo, foram analisados oito fragmentos $(\mathrm{F})$ interligados a um corredor de vegetação $(\mathrm{C})$, composto por um eixo principal e quatro ramificações (Figura 1), onde sete dos fragmentos apresentam curso de água em seu interior. A área total dos fragmentos é de 51,1624 ha, variando de 1,0302 ha o menor e 12,40 ha o maior (Tabela 1). Os corredores, abordados no presente estudo, são definidos como estruturas lineares de vegetação, de várias origens, de largura reduzida (entre 3 e 6 $\mathrm{m})$, podendo estar conectados a fragmentos de áreas variadas (Castro, 2004). O histórico de ocupação e relacionamento da população com o ambiente em que está inserida encontra-se refletido na paisagem atual, onde se percebem um número reduzido de remanescentes florestais, extensas áreas de monoculturas de gêneros alimentícios e de criação de gado. A estrutura agrária da região do Alto Rio Grande encontra-se extremamente fragmentada, o que propiciou, e ainda propicia, a formação de uma extensa malha de corredores de vegetação nativa. Basicamente existem duas origens distintas destes corredores: (i) as faixas estreitas de vegetação deixadas nos limites das propriedades após o corte raso das florestas e (ii) a colonização pela vegetação nativa dos valos, cercas e muros de pedra utilizados como divisórias entre glebas de terra. Os valos, provavelmente devido à maior umidade do solo, são naturalmente colonizados por espécies arbóreas das florestas estacionais e de galeria da região formando corredores de vegetação florestal. Esses valos divisores de glebas de terra são extremamente comuns em toda a região estudada. A vegetação dos valos, na região Sul de Minas, é conhecida popularmente como restinga. Segundo as comunidades tradicio- nais, os valos foram construídos por escravos no período da colonização e possuíam, aproximadamente, dimensões de 1,5 $\mathrm{m}$ de largura com a profundidade de $1 \mathrm{~m}$. Essas estruturas eram construídas nas propriedades onde não havia a disponibilidade de pedras para a construção de muros, respeitando-se, assim, as características da região. Por outro lado, os valos podem favorecer o aparecimento de voçorocas nas propriedades, por serem um canalizador de água, podendo ainda dificultar a passagem de animais, de veículos, de máquinas agrícolas, de linhas de irrigação e construção de curvas de níveis (Castro, 2004).

$\mathrm{Na}$ área estudada (Figura 1), podem ser identificados:

a) uma área matricial (M), composta por culturas anuais (milho, soja, feijão, dentre outras), pastagens plantadas (Brachiaria spp.) para criação de gado, campos naturais e antropizados;

b) corredores de vegetação arbórea ocorrendo em valos;

c) porções de hábitat do tipo mosaico, compreendendo vegetação de cerrado sentido amplo, matas de galerias e manchas de florestas estacionais semideciduais (Castro, 2004).

O clima da região pode ser definido, como do tipo Cwa de Köppen, com precipitação média anual de $1.529,7 \mathrm{~mm}$ e temperatura média anual de $19,4^{\circ} \mathrm{C}$ (Brasil, 1992; Ometto, 1981). A altitude mínima na área de estudo é de 920 m e a máxima de 1.180 m (Castro, 2004).

\section{Avaliação da avifauna}

\section{Levantamento de avifauna}

A amostragem da comunidade de aves foi realizada nos fragmentos, nos corredores e na matriz, a partir do crepúsculo matutino, período de maior atividade das aves diurnas e também no período crepuscular. 


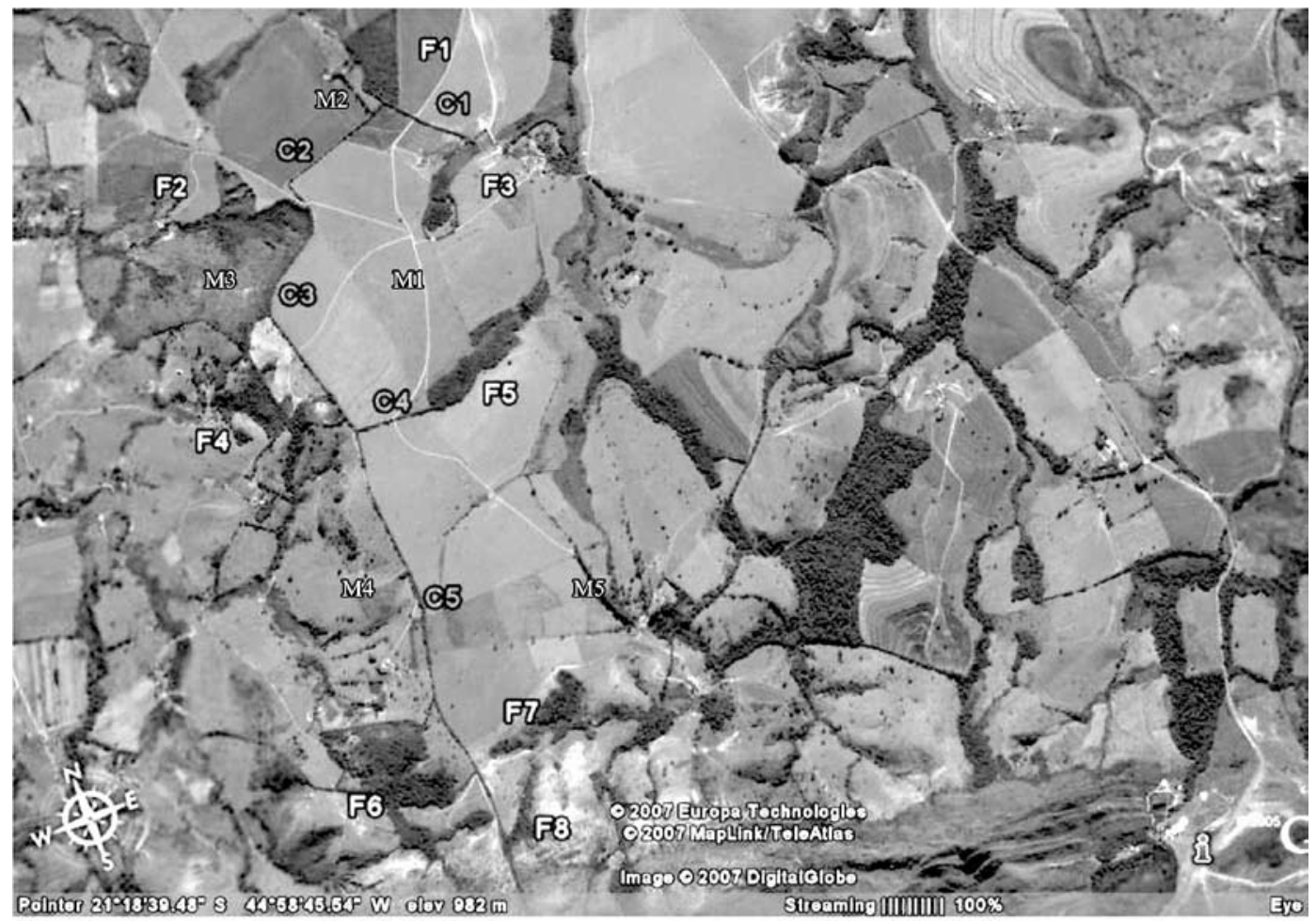

Figura 1. Mapa da área de estudo, município de Lavras - Minas Gerais, na base da Serra do Carrapato (Fonte: http://earth.google.com).

Tabela 1 - Áreas estudadas, dentro dos ambientes, com os pontos amostrados.

\begin{tabular}{|c|c|c|c|c|c|}
\hline Ambientes & $\begin{array}{l}\text { Áreas } \\
\text { (ha) }\end{array}$ & $\begin{array}{c}\text { Pontos } \\
\text { amostrados }\end{array}$ & $\begin{array}{c}\text { Área amostral } \\
\left(\mathrm{m}^{2}\right)\end{array}$ & Altitude & Coordenadas \\
\hline \multicolumn{6}{|l|}{ Fragmentos } \\
\hline $\mathrm{F} 1$ & 7,1997 & 8 & $2513 \mathrm{~m}^{2}$ & $965 \mathrm{~m}$ & $21^{\circ} 17^{\prime} 28$ ' $\mathrm{S} / 44^{\circ} 59{ }^{\prime} 13$ ', W \\
\hline $\mathrm{F} 2$ & 11,8452 & 8 & $2513 \mathrm{~m}^{2}$ & $971 \mathrm{~m}$ & $21^{\circ} 17^{\prime} 43^{\prime}, \mathrm{S} / 44^{\circ} 59,40$ ', W \\
\hline $\mathrm{F} 3$ & 1,0302 & 8 & $2513 \mathrm{~m}^{2}$ & $977 \mathrm{~m}$ & $21^{\circ} 17^{\prime} 51$ ', $\mathrm{S} / 44^{\circ} 59^{\prime} 13^{\prime \prime} \mathrm{W}$ \\
\hline $\mathrm{F} 4$ & 7,3661 & 8 & $2513 \mathrm{~m}^{2}$ & $973 \mathrm{~m}$ & $21^{\circ} 18^{\prime} 08^{\prime}, \mathrm{S} / 44^{\circ} 59^{\prime} 48^{\prime,} \mathrm{W}$ \\
\hline F5 & 7,8013 & 8 & $2513 \mathrm{~m}^{2}$ & $977 \mathrm{~m}$ & $21^{\circ} 18^{\prime} 13$ ', $\mathrm{S} / 44^{\circ} 59^{\prime} 16^{\prime \prime} \mathrm{W}$ \\
\hline $\mathrm{F} 6$ & 12,4011 & 8 & $2513 \mathrm{~m}^{2}$ & $996 \mathrm{~m}$ & $21^{\circ} 19^{\prime} 01^{\prime}, \mathrm{S} / 44^{\circ} 59^{\prime} 47^{\prime}, \mathrm{W}$ \\
\hline F7 & 2,2593 & 8 & $2513 \mathrm{~m}^{2}$ & $990 \mathrm{~m}$ & $21^{\circ} 19$ '01', $\mathrm{S} / 44^{\circ} 59^{\circ} 47^{\prime}, \mathrm{W}$ \\
\hline F8 & 1,2595 & 8 & $2513 \mathrm{~m}^{2}$ & $1044 \mathrm{~m}$ & $21^{\circ} 19^{\prime} 13^{\prime}, \mathrm{S} / 44^{\circ} 59^{\prime} 32^{\prime \prime} \mathrm{W}$ \\
\hline \multicolumn{6}{|l|}{ Corredores } \\
\hline $\mathrm{C} 1$ & 0,2 & 8 & $628 \mathrm{~m}^{2}$ & $960 \mathrm{~m}$ & $21^{\circ} 17^{\prime} 39$ ''S/44 ${ }^{\circ} 59$ '10',W \\
\hline $\mathrm{C} 2$ & 0,28 & 8 & $628 \mathrm{~m}^{2}$ & $985 \mathrm{~m}$ & $21^{\circ} 17^{\prime} 38^{\prime}, S / 44^{\circ} 59{ }^{\prime} 24^{\prime} \mathrm{W}$ \\
\hline $\mathrm{C} 3$ & 0,44 & 8 & $628 \mathrm{~m}^{2}$ & $996 \mathrm{~m}$ & $21^{\circ} 17^{\prime} 58^{\prime}, \mathrm{S} / 44^{\circ} 59^{\prime} 41^{\prime}, \mathrm{W}$ \\
\hline $\mathrm{C} 4$ & 0,12 & 8 & $628 \mathrm{~m}^{2}$ & $991 \mathrm{~m}$ & $21^{\circ} 18$ '17''S/44 ${ }^{\circ} 59^{\prime} 29^{\prime}, W$ \\
\hline $\mathrm{C} 5$ & 0,72 & 8 & $628 \mathrm{~m}^{2}$ & $1002 \mathrm{~m}$ & $21^{\circ} 18$ '43', $\mathrm{S} / 44^{\circ} 599^{\prime} 35^{\prime} \mathrm{W}$ \\
\hline \multicolumn{6}{|l|}{ Matriz } \\
\hline M1 & & 8 & $10053 \mathrm{~m}^{2}$ & $985 \mathrm{~m}$ & $21^{\circ} 17^{\prime} 46^{\prime} ' \mathrm{~S} / 44^{\circ} 59{ }^{\prime} 23$ ', W \\
\hline M2 & & 8 & $10053 \mathrm{~m}^{2}$ & $980 \mathrm{~m}$ & $21^{\circ} 17^{\prime} 33^{\prime} ' \mathrm{~S} / 44^{\circ} 59$ '28',W \\
\hline M3 & & 8 & $10053 \mathrm{~m}^{2}$ & $997 \mathrm{~m}$ & $21^{\circ} 17^{\prime} 50^{\prime} ' \mathrm{~S} / 44^{\circ} 599^{\prime} 43^{\prime}, \mathrm{W}$ \\
\hline M4 & & 8 & $10053 \mathrm{~m}^{2}$ & $934 \mathrm{~m}$ & $21^{\circ} 18,33$ ', $\mathrm{S} / 44^{\mathrm{o}} 59^{\prime} 53^{\prime \prime} \mathrm{W}$ \\
\hline M5 & & 8 & $10053 \mathrm{~m}^{2}$ & $1022 \mathrm{~m}$ & $21^{\circ} 18 ' 49$ ' $\mathrm{S} / 44^{\circ} 59$ '19',W \\
\hline $\begin{array}{l}\text { Total } \\
\text { fragmentos }\end{array}$ & 51,16 & 64 & $20104 \mathrm{~m}^{2}$ & & \\
\hline $\begin{array}{l}\text { Total } \\
\text { corredores }\end{array}$ & 1,76 & 40 & $3140 \mathrm{~m}^{2}$ & & \\
\hline Total matriz & 15,7 & 40 & $50265 \mathrm{~m}^{2}$ & & \\
\hline Total & 68,62 & 144 & $73509 \mathrm{~m}^{2}$ & & \\
\hline
\end{tabular}




\section{a) Amostragem}

Foi realizado o método qualiquantitativo de amostragem por pontos (Blondel et al., 1970; Vielliard \& Silva, 1989).

As observações foram realizadas três vezes por semana entre fevereiro a dezembro de 2005, contando com um total de 132 dias (792 horas) de trabalho de campo. Foram utilizados binóculos Nixon Action 8x40 mm. O trabalho se iniciava às 5:30 a.m., terminando por volta das 8:30 horas e entre 16:00 e 19:00). A documentação das espécies foi feita através de fotografias, com máquina fotográfica Fuji FinePix 5100S e gravações em fitas K7, utilizando-se gravador Panasonic RQ-L31.

\section{b) Amostragem nos fragmentos florestais}

Nos oito fragmentos encontrados no sistema corredor-fragmento, que serviram de área de estudo de outra dissertação (Castro, 2004), foram georreferenciados, sistematicamente, 64 pontos de observação (oito pontos em cada fragmento), sendo quatro pontos (eqüidistantes) nas bordas e quatro pontos (eqüidistantes), no interior de cada fragmento. A distância entre cada ponto foi de $40 \mathrm{~m}$. Cada ponto de observação foi coberto num raio de 10 metros, totalizando $314,1 \mathrm{~m}^{2}$ por ponto. Foram realizadas paradas de 10 minutos em cada ponto onde foram anotadas as datas das amostragens, horário, família, espécie, tipo de contato estabelecido (visual e/ou auditivo), número de contatos, comportamento (forrageio, vôo, corte), local e outras observações gerais sobre a espécie em questão.

\section{c) Amostragem dos corredores de vegetação:}

Foram feitos caminhamentos ao longo dos cinco corredores presentes no sistema corredor-fragmento, que serviram de área de estudo de outra disertação (Castro, 2004). Foram realizadas paradas de 10 minutos, para registro e fotografia de representantes de avifauna ob- servados, em oito pontos eqüidistantes em cada corredor, totalizando 40 pontos. Cada ponto de observação foi coberto num raio de 10 metros, totalizando $314,1 \mathrm{~m}^{2}$ por ponto. A distância entre cada ponto foi de $40 \mathrm{~m}$. Cada ponto marcado para o corredor, foi coberto em um raio de 10 $\mathrm{m}$, totalizando $314,1 \mathrm{~m}^{2}$ por ponto.

\section{d) Amostragem na matriz}

$\mathrm{O}$ ambiente de matriz foi dividido em cinco áreas (M1 a M5). Foram georreferenciados, sistematicamente, 40 pontos de observação (oito pontos em cada área). A distância entre cada ponto foi de $200 \mathrm{~m}$. Cada ponto de observação foi coberto num raio de 20 metros. Foram realizadas paradas de 10 minutos em cada ponto onde foram anotadas as datas das amostragens, horário, família, espécie, tipo de contato estabelecido (visual e/ou auditivo), número de contatos, comportamento (forrageio, vôo, corte), local e outras observações gerais sobre a espécie em questão.

\section{e) Levantamento quantitativo - Método de Amostragem por Pontos}

Esse método permite definir parâmetros populacionais como o índice pontual de abundância das espécies (IPA), índice de similaridade, para posterior comparação com espécies encontradas em outras áreas. Os pontos de amostragem quantitativa foram registrados em GPS para posterior incorporação ao banco de dados georreferenciados. As vocalizações foram gravadas em fitas K-7, em gravador Panasonic RQ-L31, com microfone. A identificação das espécies e a nomenclatura utilizada efetuaram-se através de referências básicas sobre aves encontradas em CBRO (2007).

\section{Descrição e caracterização da comunidade}

Para as análises de riqueza estimada de espécies, foi usado um método não-paramétri- 
co do tipo Jacknife (de primeira ordem). Esse estimador se baseia no número de espécies que ocorrem somente em uma amostra (L). É uma técnica para reduzir a subestimação do verdadeiro número de espécies em uma comunidade, com base no número representado em uma amostra, reduzindo a inclinação da ordem 1/m (Krebs, 1989; Palmer, 1990). A análise foi feita com o auxílio do programa Estimate $\mathrm{S}$ (Colwell, 2005).$$
\text { Jack } 1=\mathbf{S}+\mathbf{L} \cdot \underline{\mathbf{m}-\mathbf{1}}
$$

$\mathrm{S}=$ número de espécies observadas $\mathbf{m}$

$\mathrm{L}=$ número de espécies registradas em uma amostra

$\mathrm{m}=$ número de amostras

$\mathrm{m}-1$ = número de amostras -1
}

As estimativas de riqueza e as curvas de acumulação de espécies foram realizadas utilizando-se o programa Estimate S (Colwell, 2005), com auxílio do programa Excel 2003 e Statistica 6.0, na plataforma Windows XP. Os cálculos foram realizados com base em 500 aleatorizações (runs), considerando uma amostragem como o esforço de campo total para os ambientes avaliados (14 amostras). O intervalo de confiança da riqueza estimada foi determinado pela sequinte equação (Zar, 1999):

$$
\underset{\text { onde: }}{\text { IC }_{(\mathbf{9 5 \% )}}}=\mathbf{X} \pm \mathbf{t}_{(\boldsymbol{\alpha}, \mathrm{gl})} \mathbf{S}_{\mathbf{x}}
$$

- IC (95\%): intervalo de confiança da riqueza estimada pelo método Jacknife 1;

- X: riqueza estimada pelo método Jacknife 1;

- t $(\alpha, g l)$ : valor de $\mathrm{t}$ da distribuição de Student $(\alpha=5 \%$ para teste bicaudal);

- Sx : erro padrão da riqueza estimada.

Foi calculada uma medida de similaridade entre as áreas amostradas, representado pelo índice de similaridade de Jaccard (IJ):

$$
\mathbf{I J}=\frac{\mathbf{c}}{\mathbf{a}+\mathbf{b}+\mathbf{c}} * 100
$$

$\mathrm{c}=$ número de espécies em comum das duas comunidades comparadas

a e $\mathrm{b}=$ número de espécies exclusivas de cada uma das duas comunidades comparadas

Também foi calculado o índice de diversidade de Shannon-Wiener (H'), segundo Magurran (1988), para a comunidade. Para a obtenção desse, é importante não só o número de espécies na área, mas também a sua abundância:

$$
H^{\prime}=-\Sigma \text { Piln (Pi) }
$$

$\mathrm{Pi}=$ proporção dos indivíduos da espécie i em relação ao número total de indivíduos da comunidade

Foram realizadas Análises de Agrupamento Hierárquico (cluster) de similaridade entre ambientes, a partir de dados de abundância de espécies. O método de agrupamento foi o de UPGMA e o índice de similaridade de Bray Curtis.

\section{RESULTADOS E DISCUSSÃO}

No sistema de fragmentos/corredores/ matriz foram registradas 179 espécies de aves pertencentes a 45 famílias. Por se tratar de uma região de transição entre biomas (Cerrado e Mata Atlântica) foram observadas espécies residentes e migratórias de ambos os biomas. Os mosaicos vegetacionais presentes na região parecem facilitar a ocorrência de alguns grupos, de forma que foi possível observar as espécies em diferentes freqüências nos períodos reprodutivos e não reprodutivos.

Do total de espécies registradas, 115 espécies são típicas de áreas abertas $(64,10 \%)$ e 64 espécies são semidependentes (35,90\%), especialistas ou não (podem utilizar outros tipos de ambientes). O predomínio de certas famílias, como Tyrannidae (25 espécies) (14\%), Emberezidae (14 espécies) (7,8\%), Thraupidae (13 espécies) (7,2\%) e Trochilidae (12 espécies) $(6,7 \%)$, sugere a adequação delas aos mosaicos vegetacionais presentes na 
área e tipicamente caracterizados como áreas com grau moderado a elevado de distúrbio (D`Ângelo Neto et al., 1998).

Das 38 espécies essencialmente florestais registradas, $15(39,5 \%)$ foram observadas também nos corredores de vegetação, o que sugere que elas utilizam esses corredores para deslocamento entre os fragmentos. Estudos relacionando a importância de corredores ecológicos destacam que esses ambientes favorecem a movimentação de espécies de aves agressivas (generalistas), dificultando o deslocamento de espécies florestais (Catterall et al., 1991).

A diversidade da comunidade de aves, no sistema de mosaico dos fragmentos-corredores, pode estar relacionada a parâmetros como a qualidade do habitat e a conexão desses ambientes com corredores ecológicos. Os ambientes fragmentados favorecem espécies, mais adaptadas às pressões antrópicas, a utilizarem corredores ecológicos (Dário \& Almeida, 2000).

A análise de cluster (Figura 2) formou três grupos distintos, um deles formado pela matriz, outro pelos corredores, outro pelos fragmentos. Os ambientes de matriz (M1 a M5) foram $100 \%$ similares entre eles, mas bastante dissimilares aos outros ambientes (corredores e fragmentos). Por se tratar de ambiente aberto composto por áreas de pastagens, cultivos agrícolas e campos sujos permitem o deslocamento de diversas espécies de aves observadas. Grande parte da diversidade observada ficou registrada nesses ambientes, de forma que se pode sugerir que ambientes antropizados são favoráveis para grupos de aves pouco sensíveis, seja com dieta generalista, seja com dieta específica, mas de fácil obtenção (é o caso de insetívoros). A baixa similaridade observada entre a matriz e fragmentos $(38 \%)$ e entre matriz e corredores $(38 \%)$ pode estar relacionada a alguns fatores como a amplitude dos nichos, a estrutura do ambiente, a presença de habitats preferenciais, entre outros pois espécies exclusivas de áreas abertas reduzem a similaridade entre ambientes (Sttoufer \& Bierregaard Junior, 1995a,b).

O grupo formado pelos corredores confirmou tal resultado, possivelmente devido à similaridade florística dos fragmentos florestais (Castro, 2004), que favorece os mesmos grupos de aves que utilizam os corredores para se deslocar entre fragmentos. A similaridade entre corredores ficou entre $67 \%(\mathrm{Cl}$ e os demais corredores) a $78 \%$ (C2 e $\mathrm{C} 3)$.

Já o grupo formado pelos fragmentos apresentou maior similaridade entre os fragmentos 4 e $6(76 \%)$ em relação aos demais (60\% na média). Esses dois fragmentos supracitados são delimitados por área de campo sujo, que parece favorecer o deslocamento de espécies de aves entre fragmentos. Nesses ambientes de campo sujo adjacentes (matriz) foram observados vários registros de espécies se deslocando da área aberta para a borda dos fragmentos.

A persistência de populações em paisagens fragmentadas é dependente de alguns fatores como a permeabilidade da matriz, o grau de conectividade das paisagens com modelos de metapopulações (Wiens et al., 1997; Hanski, 1998; Moilanen \& Hanski, 1998; Colli et al., 2003) e ainda da diversidade de nichos (MacArthur, 1972). Entre esses, a permeabilidade da matriz (Antas, 1999) parece ter favorecido a ocorrência de alguns grupos de aves, em detrimento de outros. Essa permeabilidade está principalmente relacionada à eficiência de stepping stones, que geralmente funciona em graus intermediários de resistência da matriz (Antas, 1999; Uezu, 2002, 2006; Uezu et al., 2005).

O tamanho do fragmento não foi um fator que aumentasse a similaridade entre os ambientes analisados na área de estudo, pois um fragmento muito pequeno (menor que 10 ha), pode não possuir habitats suficientes para a persistência de populações de determinadas espécies e apresente características mais ho- 
mogêneas (Colli et al., 2003). Em estudo anterior, no mesmo conjunto de fragmentos-corredor, foi observada alta similaridade florística entre ambientes de borda/corredor e fragmentos (Castro, 2004), o que evidencia uma certa homogeneidade em termos de estrutura, para o estabelecimento de certos grupos de aves. $\mathrm{Na}$ verdade, outro fator que pode estar relacionado é a dificuldade de dispersão das espécies florestais (Uezu et al., 2005, Simon, 2006).

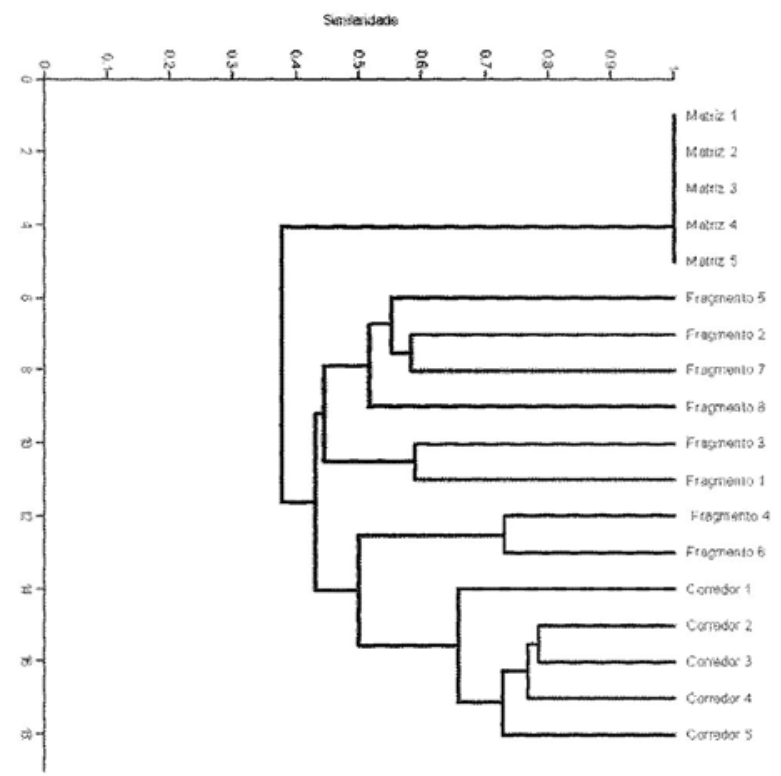

Figura 2. Dendrograma de similaridade de Bray Curtis (UPGMA) da composição da avifauna, no mosaico fragmento-corredor na fazenda da Lage, município de Lavras, Minas Gerais.

A diferença entre o índice de diversidade da avifauna (H') (Tabela 2) para fragmentos $\left(\mathrm{H}^{\prime}=3.42\right)$ e corredores $\left(\mathrm{H}^{\prime}=3.23\right)$ apresentou análises não significativas $(p<0,01)$. Assim como as relações entre o índice de diversidade para fragmentos e matriz $\left(\mathrm{H}^{\prime}=2.22\right)$ e para corredores e matriz $\left(\mathrm{H}^{\prime}=2.22\right)(\mathrm{p}>0,01)$. Os dados de similaridade total para matrizes foi mais elevado $\left(H^{\prime}=3,42\right)$, entretanto foram escolhidos e analisados os dados separados, de forma a especular as diferenças deles de acordo com a permeabilidade da matriz. D'Ângelo Neto et al. (1998) encontraram resultados mais eleva- dos de diversidade para fragmentos florestais $\left(H^{\prime}=3,55\right.$ a 3,73). Esses índices elevados, em ambientes fragmentados e reduzidos podem ser resultantes do aparecimento de espécies de aves generalistas (Lynch \& Whigham, 1984). O índice de diversidade, para estudos em área de Mata Atlântica, geralmente obtém resultado um pouco mais elevado, como os observados por Marsden et al. (2001) $\left(\mathrm{H}^{\prime}=3,93 \mathrm{em}\right.$ área de reserva e $\mathrm{H}^{\prime}=3,58$ em fragmentos florestais) e Penteado (2006) $\left(\mathrm{H}^{\prime}=3,70\right.$ a 4,3) que trabalharam em fragmentos florestais bem maiores que os estudados.

A análise da riqueza de espécies da avifauna (Jacknife 1) também pode ser observada na tabela 2. A matriz, quando avaliada no aspecto total apresentou resultado mais elevado (Jack $1=146,25)$. Entretanto, quando foram analisados os dados separadamente, os ambientes de matriz apresentaram as menores dados de riqueza (Jack $1=16,03$ a Jack $1=$ 38,90). A comparação entre a riqueza de espécies entre fragmentos florestais e corredores não apresentou diferenças significativas. Entretanto, ao se comparar a riqueza entre fragmentos florestais $\mathrm{x}$ matriz e corredores $\mathrm{x}$ matriz observaram-se diferenças significativas (Figura 3). Essas análises sugerem a influência de áreas de campo sujo adjacentes, que favorecem o deslocamento de espécies de áreas abertas para os fragmentos florestais. Parâmetros como a permeabilidade da matriz parece ser um fator que reduz o estabelecimento de espécies florestais (Simon, 2006).

Os resultados da riqueza de espécies, observados para os fragmentos 1 e 8 , sugerem que entre outros fatores, a estrutura e o tamanho dos fragmentos não estão adequadas ao estabelecimento, ou deslocamento de espécies de aves. A regressão linear realizada com $\mathrm{a}$ área dos fragmentos $\mathrm{x}$ número de indivíduos $(\mathrm{r} 2=0,13 ; \mathrm{F}=0,960 ; \mathrm{p}<0,3649)$ e área dos fragmentos $\mathrm{x}$ riqueza observada $(\mathrm{r} 2=0,23$; $\mathrm{F}=1,861 ; \mathrm{p}<0,2213)$ não foi estatisticamente significativa, indicando que o tamanho da 
área não influencia na distribuição das espécies de aves para o sistema estudado. Estudos relacionando aves e sua distribuição em ambientes florestais geralmente são realizados em fragmentos maiores (100 ha, 200 ha, 300 ha ou maiores) seja na Floresta Atlântica (Ribon, 2003; Anjos, 2001; Develey, 2004) ou na Floresta Amazônica (Stouffer
\& Bierregaard Junior, 1995a,b; Laurance \& Bierregaard Junior, 1997). Uma hipótese proposta por Lomolino (2000) destaca que, para áreas muito pequenas, não é possível evidenciar a relação espécie-área e quais aspectos envolvem essa relação, sejam efeitos demográficos de populações pequenas ou efeitos estocásticos.

Tabela 2. Índice de diversidade ( $\left.\mathrm{H}^{\prime}\right)$ e Riqueza de espécies (Jacknife) da avifauna, na área de estudo.

$\begin{array}{ccccccccc}\text { Ambientes } & \mathbf{N}^{\mathbf{0}} \text { espécies } & \mathbf{N}^{\mathbf{0}} \text { indivíduos } & \text { ID } & \text { Jack 1 } & \text { DP } & \text { I.C. (95\%) } & \text { H' } & \text { DP } \\ \text { Fragmento 1 } & 38 & 231 & 117.857 & 48.63 & 2.27 & 1.73 & 3.26 & 0 \\ \text { Fragmento 2 } & 59 & 341 & 173.98 & 78.38 & 2.94 & 2.82 & 3.69 & 0 \\ \text { Fragmento 3 } & 56 & 335 & 170.918 & 70 & 5.77 & 2.57 & 3.53 & 0 \\ \text { Fragmento 4 } & 50 & 234 & 119.388 & 64.88 & 3.61 & 2.36 & 3.48 & 0 \\ \text { Fragmento 5 } & 50 & 197 & 100.51 & 65.75 & 4.13 & 2.42 & 3.54 & 0 \\ \text { Fragmento 6 } & 47 & 209 & 106.633 & 63.75 & 3.91 & 2.31 & 3.22 & 0 \\ \text { Fragmento 7 } & 44 & 162 & 82.653 & 58.88 & 3.84 & 2.13 & 3.46 & 0 \\ \text { Fragmento 8 } & 33 & 125 & 63.776 & 44.38 & 3.95 & 1.65 & 3.22 & 0 \\ \text { Corredor 1 } & 42 & 406 & 207.143 & 52.63 & 3.73 & 1.93 & 3.1 & 0 \\ \text { Corredor 2 } & 43 & 367 & 187.245 & 52.63 & 1.84 & 1.91 & 3.13 & 0 \\ \text { Corredor 3 } & 44 & 315 & 160.714 & 57.38 & 2.94 & 2.14 & 3.31 & 0 \\ \text { Corredor 4 } & 40 & 292 & 148.98 & 47.88 & 2.45 & 1.76 & 3.2 & 0 \\ \text { Corredor 5 } & 49 & 313 & 159.694 & 57.75 & 2.19 & 2.14 & 3.41 & 0 \\ \text { Matriz 1 } & 49 & 378 & 199.995 & 22.97 & 0.93 & 2.44 & 2.14 & 0 \\ \text { Matriz 2 } & 47 & 347 & 182.604 & 20.95 & 1.42 & 2.34 & 2.26 & 0 \\ \text { Matriz 3 } & 39 & 683 & 339.122 & 38.90 & 2.24 & 4.21 & 2.70 & 0 \\ \text { Matriz 4 } & 42 & 293 & 147.822 & 16.96 & 1.34 & 1.93 & 2.10 & 0 \\ \text { Matriz 5 } & 50 & 276 & 139.030 & 16.03 & 1.25 & 1.84 & 1.93 & 0\end{array}$

$\mathrm{H}^{\prime}=1,0$ a 2,0 (avifauna de florestas temperadas)

$\mathrm{H}^{\prime}=3,0$ (avifauna de ambientes tropicais) Vielliard \& Silva (1989)

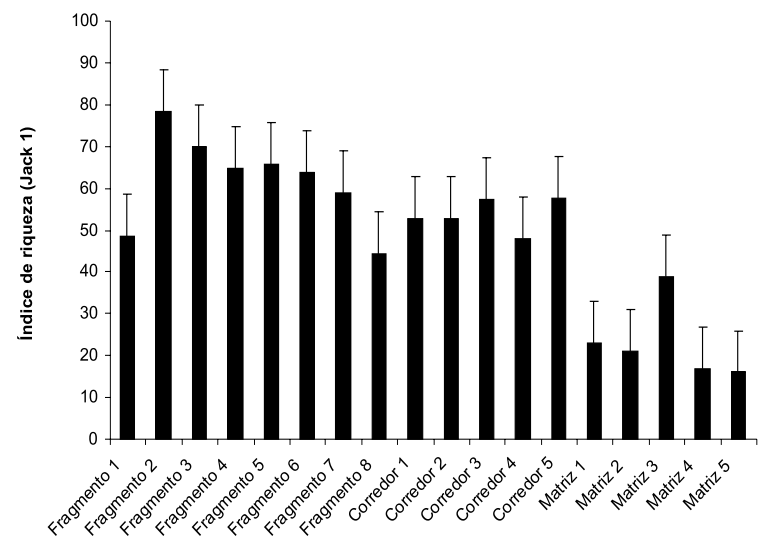

Figura 3. Gráfico geral do índice de riqueza (Jack 1) da avifauna amostrada no mosaico fragmento-corredor na fazenda da Lage, município de Lavras, Minas Gerais.
As características da área estudada, envolvendo o histórico de ocupação e a presença de remanescentes florestais semideciduais interligados por corredores, possibilita a presença de uma variedade de habitats (Anjos et al., 2004; Penteado, 2006). Segundo Uezu et al. (2005), o tamanho do fragmento exerce papel fundamental para explicar variações na riqueza e composição de espécies, em ambientes com baixa conectividade, enquanto que, em paisagens bem conectadas, a influência da matriz é mais importante do que o tamanho da área. 


\section{CONCLUSÃO}

A distribuição de aves no complexo estudado parece ser mais afetada pela estrutura do que pelo tamanho dos fragmentos. É possível que exista influência na distância entre fragmentos para a distribuição de grupos florestais dependentes e semidependentes de aves, mas isso não foi avaliado. Houve predomínio de grupos generalistas e de áreas abertas. Esse fator sugere que certos grupos de avifauna apresentam preferência de habitat. Os grupos florestais semidependentes são favorecidos pela permeabilidade da matriz, em relação aos grupos dependentes.

A baixa riqueza de espécies exclusivamente florestais indica que a área de estudo é similar a uma colcha de retalhos, e parece que os fragmentos não fornecem nichos, a estrutura do ambiente, a presença de habitats preferenciais suficientes para suportar mais espécies florestais do que foi registrado.

Os corredores funcionaram de maneira eficiente para o deslocamento de aves e para descanso. A riqueza de espécies semidependentes que utilizam os corredores realmente necessitam dessa estrutura para se deslocar entre os fragmentos. Como existem vários corredores conectando os fragmentos, tal sistema pode favorecer a dinâmica da taxocenose de aves.

São necessários estudos a longo prazo em ambientes fragmentados, correlacionando sempre alterações nas matrizes adjacentes e suas implicações na dinâmica da comunidade avifaunística.

\section{REFERÊNCIAS BIBLIOGRÁFICAS}

ANJOS, L. Bird communities in five atlantic forest fragments in southern Brazil. Ornitologia Neotropical, [S.1.], v. 12, p. 11-27, 2001.

ANJOS, L.; ZANETTE, L.; LOPES, E. V. Effects of fragmentation on the bird guilds fo the atlantic forest in north Paraná, southern Brazil. Ornitologia Neotropical, [S.1.], v. 15, p. 137-144, 2004.

ANTAS, P. T. Z. Comunidades de aves dos cerrados do Planalto Central e porção ocidental do médio rio São Francisco. 1999. Tese (Doutorado em Ecologia) - Universidade de Brasília, Brasília, DF.

ANTUNES, A. Z. Alterações na composição da comunidade de aves ao longo do tempo em um fragmento florestal no sudeste do Brasil. Revista Brasileira de Ornitologia, Ararajuba, v. 13, n. 1, p. 47-61, Jun. 2005.

BIRDLIFE INTERNATIONAL. Lynx edicions e birdlife international. [S.1.], 2000. $852 \mathrm{p}$.

BLONDEL, J.; FERRY, C.; FROCHOT, B. La méthode des indices ponctuels d'abundance (IPA) ou des relevés d'avifaune par "stations d'écoute". Alauda, [S.1.], v. 38, p. 55-71, 1970.

BRASIL. Ministério da Agricultura. Departamento Nacional de Meteorologia. Normas climatológicas: 1961-1990. Brasília, DF, 1992. $132 \mathrm{p}$.

CASTRO, G. C. Análise da estrutura, diversidade florística e variações espaciais do componente arbóreo de corredores de vegetação na região do Alto Rio Grande, MG. 2004. 83 f. Dissertação (Mestrado em Manejo Ambiental).

CATTERALL, C. P.; GREEN, R. J.; JONES, D. N. Habitat use by birds across a forestsuburb interface in Brisbane: implications for corridors. In: SAUNDERS, D. A.; HOBBS, R. J. (Eds.). Nature conservation 2: the role of corridors. New South Wales: Surrey Beatty \& Sons, 1991. p. 247-258. 
COLLI, G. R.; ACCACIO, G. M.; ANTONINI, Y.; CONSTANTINO, R.; FRANCESCHINELLI, E. V.; LAPS, R. R.; SCARIOT, A.; VIEIRA, M. V.; WIEDERHECKER, H. C. A fragmentação dos ecossistemas e a biodiversidade brasileira: uma síntese. In: RAMBALDI, D. M.; OLIVEIRA, D. A. S. de (Orgs.). Fragmentação de ecossistemas. Brasília, DF: Ministério do Meio Ambiente, 2003. v. 1, p. 317-324.

COLWELL, R. K. Estimates: statistical estimation of species richness and shared species from samples. Version 7.5. 2005. Disponível em: <http://purl.oclc.org/estimates $>$. Acesso em: 1 dez. 2007.

COMITÊ BRASILEIRO DE REGISTROS ORNITOLÓGICOS. Listas das aves do Brasil. Versão 16.8/2007. Disponível em: <http:// www.cbro.org.br>. Acesso em: 20 jan. 2007.

D'ANGELO NETO, S.; VENTURIN, N.; OLIVEIRA-FILHO, T. A.; COSTA, F. A. F. Avifauna de quatro fisionomias florestais de pequeno tamanho (5-8 ha) no campus da UFLA. Revista Brasileira de Biologia, São Paulo, v. 58, n. 3, p. 463-472, 1998.

DÁRIO, F. R.; ALMEIDA, A. F. Influência de corredor florestal sobre a avifauna da Mata Atlântica. Scientia Forestalis, Piracicaba, n. 58, p. 99-109, 2000.

DEVELEY, P. F. Efeitos da fragmentação e o estado de conservação da floresta na diversidade de aves da mata atlântica. 2004. Tese (Doutorado) - Universidade de São Paulo, São Paulo.

GROOM, J. D.; GRUBB, T. C. Patch colonization dynamics in Carolina Chickadees (Poecile carolinensis) in a fragmented landscape: a manipulative study. Auk, [S.1.], v. 123, n. 4, p. 1149-1160, 2006.
HANSKI, I. Metapopulation dynamics. Nature, [S.1.], v. 396, p. 41-49, 1998.

IBAMA. Portaria n. 1.522, de 19 de dezembro de 1.989. Brasília, DF, 1989.

IBAMA. Portaria n. 45-N, de 27 de abril de 1992. Brasília, DF, 1992.

KELLMAN, M.; TACKABERRY, R.; MEAVE, J. The consequences of prolonged fragmentation: lessons from tropical gallery forests. In: SCHELLAS, J.; GREENBERG, R. (Eds.). Forest patches in tropical landscapes. Washington, DC: University Island, 1996. p. 37-58.

KREBS, C. J. Ecological methodology. [S.1.]: Harper Collins, 1989. 654 p.

LAPS, R. R.; CORDEIRO, P. H. C.; KAJIWARA, D.; RIBON, R.; RODRIGUES, A. A. F.; UEJIMA, A. Aves. In: RAMBALDI, D. M.; D. A. S. O. (Orgs.). Fragmentação de ecossistemas: causas, efeitos sobre a biodiversidade e recomendações de políticas públicas. Brasília, DC: MMA/SBF, 2003. p. 53-181, 510 p.

LAURANCE, W. F.; BIERREGAARD JUNIOR, R. O. Tropical forest remnants: ecology, management, and conservation of fragmented communities. Chicago: University of Chicago, 1997. $616 \mathrm{p}$

LIMA, M. G. de; RAMOS NETO, M. B.; MACHADO, R. B.; MOREIRA, R. A.; CAVALCANTI, R. B. Landscape analysis and bird's eye view of habitat structure in the neighbouring areas of Emas National Park, Brazil. In: CONGRESSO DE ECOLOGIA DO BRASIL, 6., 2003, Fortaleza. Anais... Fortaleza: UFCE, 2003.

LOMOLINO, M. V. Ecology's most general, yet protean 1 pattern: the species-area rela- 
tionship. Journal of Biogeography, Sydney, v. 27, n. 1, p. 17-26, Jan. 2000.

LYNCH, J. F.; WHIGHAM, D. F. Effects of forest fragmentation on breeding bird communities in Maryland, USA. Biological Conservation, Oxford, v. 28, n. 2, p. 287-324, 1984.

MacARTHUR, R. H. Geographical ecology: patterns in the distribution of species. New York: Harper \& Row, 1972. 269 p.

MAGURRAN, A. Ecological diversity and its measurement. Cambridge: University of Cambridge, 1988. 179 p.

MALDONADO-COELHO, M.; MARINI, M. Effects of forest fragment size and sucessional slage on mixed species bird flocks in southeastern Brazil. The Condor, Santa Clara, v. 102, p. 585-594, 2000.

MARINI, M. A. Effects of forest fragmentation on birds of the cerrado region, Brazil. Bird Conservation International, [S.1.], v. 11, n. 1, p. 13-25. 2001.

MARSDEN, S. J.; WHIFFIN, M.; GALETTI, M. Bird diversity and abundance in forest fragments and Eucalyptus plantations around an Atlantic forest reserve, Brazil. Biodiversity and Conservation, London, v. 10, p. 737-751, 2001.

MOILANEN, A.; HANSKI, I. Metapopulation dynamics: effects of habitat quality and landscape structure. Ecology, Durham, v. 79, n. 7, p. 2503-2515, 1998.

NOSS, R. F.; CSUTI, B.; GROOM, M. J. Habitat fragmentation: principles of conservation biology. Sunderland: Sinauer Association, 2006. 699 p.

OMETTO, J. C. Bioclimatologia vegetal. São Paulo: Agronômica Ceres, 1981. 440 p.
PALMER, M. W. The estimation of species diversity. Annual Review of Ecology and Systematics, [S.1.], v. 5, p. 285-307, 1990.

PENTEADO, M. Distribuição e abundância de aves em relação ao uso da terra na bacia do rio Passa-Cinco, estado de São Paulo, Brasil. 2006. 132 f. Tese (Doutorado) - Escola Superior de Agricultura Luiz de Queiroz, Universidade de São Paulo, Piracicaba.

RIBON, R. Aves em fragmentos de mata atlântica do sudeste de Minas Gerais: incidência, abundância e associação topográfica. 2003. Tese (Doutorado) - Universidade Federal de Minas Gerais, Belo Horizonte.

SILVA, J. M. C. da. Biogeographic analysis of the South American Cerrado avifauna. Steenstrupia, Copenhagen, v. 21, p. 49-67, 1995a.

SILVA, J. M. C. da. Birds of the Cerrado Region, South America. Steenstrupia, Copenhagen, v. 21, n. 1, p. 69-92, 1995b.

SIMON, J. E. Efeitos da fragmentação da mata atlântica sobre comunidade de aves na região serrana de Santa Tereza, estado do Espírito Santo, Brasil. 2006. 142 f. Tese (Doutorado) - Universidade Federal do Rio de Janeiro, Rio de Janeiro.

SOARES, E. S.; ANJOS, L. Efeito da fragmentação florestal sobre aves escaladores de tronco e galho na região de Londrina, norte do estado do Paraná, Brasil. Ornitologia Neotropical, [S.1.], v. 10, n. 1, p. 61-68, 1999.

STOUFFER, P. C.; BIERREGAARD JUNIOR, R. O. Effects of fragmentation on understory hummingbirds in Amazonian Brazil. Conservation Biology, Oxford, v. 9, n. 4, p. 1085-1094, 1995b. 
STOUFFER, P. C.; BIERREGAARD, R. O. Use of Amazonian forest fragments by understory insectivorous birds. Ecology, Durham, v. 76, n. 8, p. 2429-2445, 1995a.

TUBELIS, D. P. Estrutura de comunidades de aves em habitats preservados e alterados de cerrado, na região do Distrito Federal. 1997. Dissertação (Mestrado em Ecologia) Universidade de Brasília, Brasília, DF.

UEZU, A. Composição e estrutura da comunidade de aves na paisagem fragmentada do Pontal do Paranapanema. 2006. 193 f. Tese (Doutorado) - Universidade de São Paulo, São Paulo.

UEZU, A. Efeito do tamanho do fragmento e do tipo de matriz na abundância de seis espécies de aves florestais em Caucaia do Alto, SP. 2002. 79 f. Dissertação (Mestrado em Ecologia) - Universidade de São Paulo, São Paulo.

UEZU, A.; METZGER, J. P.; VIELLIARD, J. M. E. Effects on structural and functional con- nectivity and patch size on the abundance of seven Atlantic Forest bird species. Biological Conservation, Oxford, v. 123, n. 4, p. 507$519,2005$.

VIELLIARD, J.; SILVA, W. R. Nova metodologia de levantamento quantitativo e primeiros resultados no interior do Estado de São Paulo. In: ENCONTRO NACIONAL DE ANILHADORES DE AVES, 4., 1989, Recife. Anais... Recife: UFRPe, 1989. p. 117-151.

WICKAM, J. D.; RIITTERS, K. H.; WADE, T. G.; COULTON, J. W. Temporal change in forest fragmentation at multiple scales. Landscape Ecology, [S.1.], v. 22, n. 4, p. 481-489, 2007.

WIENS, J. A.; SCHOOLEY, R. L.; WEEKS JUNIOR, R. D. Patchy landscapes and animal movements: do beetles percolate? Oikos, Copenhagen, v. 78, p. 257-264, 1997.

ZAR, J. R. Biostatistical analysis. Prentice Hall: New Jersey, 1999. 663 p. 